\title{
Estudo da produção científica sobre o enfoque CTS em revistas brasileiras especializadas
}

\author{
Study of the scientific production about the STS approach in brazilian \\ specialized journals
}

\author{
Andrei Steveen Moreno Rodríguez ${ }^{1}$ \\ José Claudio Del Pino²
}

\section{Resumo}

O estudo teve como objetivos principais: mapear, classificar e analisar descritivamente produções científicas acerca do enfoque Ciência, Tecnologia e Sociedade - CTS, publicadas entre 2011 e 2015 em revistas brasileiras. Foram selecionadas 4 revistas especializadas nas áreas de Ensino e Educação, classificadas com Qualis A1 e A2. Por meio da leitura de títulos e resumos dos 803 artigos publicados nesse período, foram escolhidas 30 publicações para serem analisadas. As principais informações bibliométricas desses textos foram sistematizadas em formato de tabela e os documentos completos dispostos em uma unidade hermenêutica do software ATLAS.ti para posterior análise. A partir de critérios bibliométricos e da leitura completa dos textos foi possível identificar: 1) interesse dos pesquisadores por trabalhar no campo da Educação em Ciências e pela formação de professores; 2) interesse pela abordagem de questões sociocientíficas; 3) tendência à realização de intervenções didáticas e estudos de caso; 4) interesse por conhecer percepções da comunidade acadêmica acerca de ciência e de tecnologia, assim como pelas dificuldades para a implementação de atividades CTS. Concluiu-se que nos últimos anos tem sido consolidado um conjunto de referências brasileiras que discutem o tema, gerando, consequentemente, uma forma particular de pensar o enfoque CTS. No entanto, percebeu-se a necessidade de aproximar essa produção com estudos realizados nos países vizinhos para, dessa forma, contribuir para a construção de uma perspectiva latino-americana de progresso educativo, social e tecnocientífico.

Palavras-chave: Produção científica; Enfoque CTS; Brasil; Educação em Ciências.

\section{Abstract}

The main objectives of the study were: to map, classify and descriptively analyze scientific productions about the Science, Technology and Society - STS approach, published between 2011 and 2015 in Brazilian journals. Four specialized journals were selected in the areas of Teaching and Education, classified as Qualis A1 and A2. By reading of titles and abstracts of the 803 articles published in that period, 30 publications were chosen to be analyzed. The main bibliometric information of these texts was systematized in table format and the complete documents were placed in a hermeneutical unit of the ATLAS.ti software for further analysis. Based on bibliometric criteria and the complete reading of the texts it was possible to identify: 1) interest of researchers in working for Science Education and

\footnotetext{
1 Universidade Estadual de Santa Cruz | moreno.rodriguez@ufrgs.br

2 Universidade Federal do Rio Grande do Sul | delpinojc@yahoo.com.br
} 
teacher training; 2) interest in addressing socio-scientific issues; 3) tendency to implement didactic interventions and case studies; 4) interest in understanding the perceptions of the academic community about science and technology, as well as the difficulties for the implementation of STS activities. It was possible to conclude that in recent years, a set of Brazilian references, which discuss the topic, has been consolidated. Consequently, a particular way of thinking about the CTS approach in the country was generated. However, it was possible to perceive the need to approximate this production with studies conducted in neighboring countries, in order to contribute to the construction of a Latin American perspective of educational, social and techno-scientific progress.

Key-words: Scientific Production; STS Approach; Brazil; Science Education.

\section{Resumen}

El estudio tuvo como objetivos principales: rastrear, clasificar y analizar descriptivamente producciones científicas acerca del enfoque Ciencia, Tecnología y Sociedad - CTS publicadas en revistas brasileras entre 2011 y 2015. Para su realización fueron seleccionadas 4 revistas especializadas en las áreas de Enseñanza y Educación, clasificadas con Qualis A1 y A2. Por medio de la lectura de los títulos y resúmenes de los 803 artículos publicados en ese periodo, fueron escogidas 30 publicaciones para ser analizadas. Sus principales informaciones bibliométricas fueron sistematizadas en formato de tabla y los documentos completos insertados en una unidad hermenéutica del software ATLAS.ti para posterior análisis. A partir de criterios bibliométricos y de la lectura completa de los textos fue identificado: 1) interés de los investigadores por trabajar en el campo de la Educación en Ciencias y en la Formación de Profesores; 2) disposición para abordar cuestiones sociocientíficas en el aula; 3) inclinación hacia la realización de intervenciones didácticas y estudios de caso; 4) interés por conocer las percepciones de la comunidad académica acerca de la ciencia y de la tecnología, así como por las dificultades para la implementación de actividades CTS. Fue posible concluir que en los últimos años se ha consolidado un conjunto de referencias brasileras que discuten el tema, generando, consecuentemente, una forma particular de pensar el enfoque CTS. Sin embargo, se notó la necesidad de aproximar esa producción con estudios realizados en los países vecinos para, de esa forma, contribuir a la construcción de una perspectiva latinoamericana de progreso educativo, social y tecnocientífico.

Palabras clave: Producción científica; Enfoque CTS; Brasil; Educación en Ciencias.

\section{Introdução}

Desde finais do século passado, uma série de estudos e reflexões acerca do papel da ciência e da tecnologia e de suas relações com a sociedade e o ambiente estão sendo desenvolvidos sob a perspectiva do denominado movimento Ciência, Tecnologia e Sociedade - CTS (AULER; BAZZO, 2001; LÓPEZ CEREZO, 1998; MARTÍNEZ ÁLVAREZ, 2004; MEMBIELA, 1997; QUINTERO, 2010; SANTOS; MORTIMER, 2002), em razão de as consequências negativas do desenvolvimento tecnocientífico descontrolado serem cada vez mais notórias.

Essas reflexões têm permeado vários campos do conhecimento, repercutindo inclusive na política, na pesquisa e na Educação. Nesse último campo, têm surgido diversas reflexões a respeito da formação científica e tecnológica que é promovida nos diversos níveis acadêmicos, e de como esta pode influenciar diretamente na formação para a cidadania e no progresso social (SANTOS, 2012). 
Assim, consolida-se o "Enfoque CTS" (denominação empregada para se referir às repercussões do movimento CTS no contexto educacional) (STRIEDER, 2012). Este, a partir da rejeição da ciência como atividade pura e neutra, da crítica à tecnologia entendida como aplicação da ciência e da reprovação da tecnocracia (LÓPEZ CEREZO, 1998), promove o interesse dos estudantes, discussões sobre as implicações éticas do desenvolvimento tecnocientífico, o desenvolvimento do pensamento crítico e a participação cidadã em assuntos relacionados com ciência e tecnologia.

No Brasil, segundo relatam diferentes autores (ABREU et al., 2013; SANTOS, 2008; STRIEDER, 2012) a produção acadêmica acerca deste enfoque sofreu um incremento nos últimos anos, trazendo uma série de abordagens e perspectivas interessantes que contribuem para a evolução deste campo de estudos.

Este trabalho apresenta uma análise da produção científica sobre o enfoque CTS em revistas brasileiras, com o intuito de responder os questionamentos a seguir: De que forma as pesquisas com esse enfoque estão sendo desenvolvidas? Que temas abordam? O que procuram? Quais os principais procedimentos metodológicos empregados para seu desenvolvimento? Que autores estão desenvolvendo esse tipo de trabalho? Que autores têm contribuído mais fortemente na construção do corpus teórico CTS no país?

Os principais objetivos foram: mapear, classificar e analisar descritivamente, em revistas científicas especializadas nas áreas de Ensino e Educação no Brasil, produções modernas que abordassem o enfoque CTS. Para tal fim foram analisados trabalhos publicados durante os cinco anos imediatamente anteriores à realização do estudo (entre 2011 e 2015).

\section{Aspectos metodológicos}

Baseado no uso de técnicas bibliométricas para levantamento de dados, o presente estudo apresenta uma descrição quantitativa e qualitativa de informações referentes à produção de artigos científicos relacionados com o enfoque CTS no Brasil.

A seleção das revistas analisadas, foi realizada com base nas informações oferecidas na Plataforma Sucupira, descrita pela CAPES (2017a) como uma "ferramenta para coletar informações, realizar análises e avaliações e ser a base de referência do Sistema Nacional de Pós-Graduação (SNPG) ".

A busca das revistas foi realizada em dezembro de 2015 a partir dos seguintes critérios:

a) Classificação de periódicos $\left(\right.$ Qualis $\left.^{3}\right)$ (2014)

b) Áreas de avaliação: Ensino e Educação

3 "O Qualis-Periódicos é um sistema usado para classificar a produção científica dos programas de pósgraduação no que se refere aos artigos publicados em periódicos científicos [...] A classificação de periódicos é realizada pelas áreas de avaliação e passa por processo anual de atualização. Esses veículos são enquadrados em estratos indicativos da qualidade - A1, o mais elevado; A2; B1; B2; B3; B4; B5; C - com peso zero". (CAPES, 2017b) 
c) Descritores: CTS; Ciência; Ciência e Sociedade; Ciência Tecnologia e Sociedade; Tecnologia; Tecnologia e Sociedade; Sociedade.

A partir dos resultados obtidos, foram selecionadas exclusivamente as revistas classificadas com Qualis A (A1 ou A2) nas duas áreas de avaliação (Ensino e Educação).

No Quadro 1 são apresentadas as revistas selecionadas. Estas são representativas para a comunidade acadêmica e possuem excelente prestigio, além de livre acesso (online).

Posteriormente procedeu-se à identificação dos artigos publicados entre 2011 e 2015 que abordassem o enfoque CTS. A seleção das publicações foi baseada na leitura atenta dos títulos e resumos do total dos artigos divulgados nas quatro revistas durante o período mencionado anteriormente. A partir desse processo foram identificados 30 artigos que efetivamente discutem o tema em questão.

Quadro 1. Revistas selecionadas para análise.

\begin{tabular}{l|c|c|c}
\hline \multicolumn{1}{c|}{ Nome } & ISSN & $\begin{array}{c}\text { Qualis } \\
2014 \\
\text { ENSINO }\end{array}$ & $\begin{array}{c}\text { Qualis 2014 } \\
\text { EDUCAÇÃO }\end{array}$ \\
\hline Ciência \& Educação & $1980-850 X$ & $\mathrm{~A} 1$ & $\mathrm{~A} 2$ \\
\hline Ensaio: Pesquisa em Educação em Ciências (Online) & $1983-2117$ & $\mathrm{~A} 2$ & $\mathrm{~A} 2$ \\
\hline Investigações em Ensino de Ciências (Online) & $1518-8795$ & $\mathrm{~A} 2$ & $\mathrm{~A} 2$ \\
\hline Revista Brasileira de Pesquisa em Educação em Ciências & $1806-5104$ & $\mathrm{~A} 2$ & $\mathrm{~A} 2$ \\
\hline
\end{tabular}

Em seguida, as principais informações bibliométricas dos 30 textos foram organizadas em formato de tabela contendo campos referentes a: revista, título, palavras-chave, link direto ao artigo, ano, volume, número, país dos autores, nomes dos autores. A partir do tratamento estatístico desses dados, foram obtidas diversas informações a serem discutidas neste trabalho.

Todos os trabalhos selecionados foram lidos na íntegra para sua análise. Os documentos completos, em formato pdf, foram organizados em uma interface do software ATLAS.ti (MUHR, 1991), que facilitou a seleção de fragmentos de texto que indicassem os temas abordados, as questões de pesquisa propostas, os tipos de procedimentos metodológicos desenvolvidos e os objetos/sujeitos analisados nesses estudos, assim como reunir informações com semelhança de significados para a criação de categorias. Também foi realizada uma caraterização do total das referências empregadas nas publicações com o intuito de identificar os autores que tem contribuído mais vigorosamente na construção teórica do campo CTS no país.

A seguir serão apresentados e discutidos os resultados desse processo.

\section{Resultados e discussão}

A Tabela 1 apresenta a quantidade total de artigos publicados entre 2011 e 2015 nas quatro revistas escolhidas, assim como a quantidade de artigos relacionados ao enfoque CTS. Estes foram selecionados para a realização das seguintes etapas do estudo. 
Tabela 1. Quantidade de artigos publicados por revista entre 2011 e 2015.

\begin{tabular}{llcccccc}
\cline { 3 - 7 } & & 2017 & 2012 & 2013 & 2014 & 2015 & Total \\
\hline \multirow{2}{*}{ Ciência \& Educação } & Artigos publicados & 60 & 60 & 60 & 60 & 60 & 300 \\
& Artigos enfoque CTS & 2 & 1 & 2 & 3 & 2 & 10 \\
\hline Ensaio: Pesquisa em Educação & Artigos publicados & 34 & 49 & 36 & 33 & 23 & 175 \\
em Ciências (Online) & Artigos enfoque CTS & 0 & 3 & 1 & 2 & 1 & 7 \\
\hline Investigações em Ensino de & Artigos publicados & 24 & 36 & 36 & 36 & 32 & 164 \\
Ciências (Online) & Artigos enfoque CTS & 0 & 0 & 2 & 1 & 1 & 4 \\
\hline Revista Brasileira de Pesquisa & Artigos publicados & 30 & 30 & 30 & 44 & 30 & 164 \\
em Educação em Ciências & Artigos enfoque CTS & 1 & 0 & 1 & 5 & 2 & 9 \\
\hline & & \multicolumn{7}{c}{ Total artigos publicados } & & 803 \\
\cline { 2 - 7 } & & Total artigos selecionados & 30
\end{tabular}

Os dados indicam que a produção acadêmica sob a perspectiva CTS em revistas especializadas nas áreas de Ensino e Educação no Brasil é ainda pouco expressiva, pois em um universo de 803 publicações, apenas 30 artigos abordaram o tema, isto é, 3,73\% da produção total.

Destaca-se a Revista Brasileira de Pesquisa em Educação em Ciências durante o ano 2014, pois incrementou sua produção de artigos sobre CTS no volume 14, n. 2, que teve o título Environmental Discourses in Science Education: contributions to democracy, citizenship and social justice, e foi desenvolvido em parceria com os editores convidados Giuliano Reis (Ottawa University, Canada) e Alandeon Oliveira (University at Albany, EUA) (MATTOS; VILLANI, 2014). A particular importância desse número é que, além de ter contribuído com maior quantidade de artigos para este estudo, foi o único que apresentou trabalhos em língua inglesa.

Tabela 2. País de atuação dos autores.

\begin{tabular}{lc}
\hline \multicolumn{1}{c}{ País } & No de autores \\
\hline Brasil & 59 \\
Portugal & 7 \\
Canadá & 3 \\
Estados Unidos & 2 \\
Austrália & 1 \\
Colômbia & 1 \\
Espanha & 1 \\
\hline Total & 74 \\
\hline
\end{tabular}

Continuando com os resultados, a Tabela 2, apresenta o país de atuação dos autores dos artigos selecionados. Com base nesses dados é possível estimar que os trabalhos foram desenvolvidos, em sua maioria, no Brasil, pois 59 autores (79,7\%) atuam em instituições brasileiras. Esse fato possibilita, para efeitos deste estudo, a descrição de caraterísticas do panorama atual das pesquisas sobre o enfoque CTS no país. 
Com relação aos autores dos trabalhos, a maioria deles possuem apenas uma publicação nestas revistas durante o período pesquisado, apenas três autores fizeram duas publicações, Cosenza Angélica, Martins Isabel e Santos Wildson. Este último autor, pesquisador reconhecido na área, é um dos pioneiros dos estudos CTS no Brasil e produtor de importantes trabalhos citados quase na maioria dos artigos analisados, como será possível observar posteriormente.

Com o intuito de constituir uma visão geral de como estão sendo desenvolvidas as pesquisas CTS no país, os resultados, a partir de agora, estarão focados no conteúdo dos artigos estudados, começando pelas palavras-chave empregadas pelos autores. A Tabela 3, apresenta as mais frequentes.

Tabela 3. Palavras-chave mais referidas nos artigos.

\begin{tabular}{lc}
\hline \multicolumn{1}{c}{ Palavras chave } & Número de ocorrências \\
\hline Ensino de Ciências & 7 \\
CTS & 6 \\
Formação de Professores & 4 \\
Ciência & 3 \\
Ensino de Química & 3 \\
Questões Sociocientíficas & 3 \\
Alfabetização Científica e Tecnológica & 2 \\
Análise Crítica do Discurso & 2 \\
Argumentação & 2 \\
Ciência, Tecnologia e Sociedade & 2 \\
Currículo & 2 \\
Educação em Ciências & 2 \\
Ensino de Biologia & 2 \\
Ensino Fundamental & 2 \\
Tecnologia & 2 \\
Temas Controversos & 2 \\
\hline
\end{tabular}

Vale ressaltar o uso de palavras como Ensino de Ciências e Formação de Professores, posto que demonstra a preocupação dos pesquisadores em contribuir para a melhoria desses dois espaços acadêmicos que se correlacionam e que são de suma importância para o progresso educacional e social de qualquer nação. Também chamam a atenção os descritores "Questões sociocientíficas" e "Temas controversos", em razão de serem considerados, nos últimos anos, como ponto de partida para colocar em prática, os pressupostos do movimento CTS em processos educativos, promovendo não apenas a aprendizagem de conteúdos científicos, mas também habilidades como argumentação, pensamento crítico, tomada de decisão e, consequentemente, a participação cidadã em ciência e tecnologia (AZEVEDO et al., 2013; COCENZA; MARTINS, 2013; MARTíNEZ PÉREZ, 2012; MARTíNEZ PÉREZ, 2014; MENDES; SANTOS, 2013). A abordagem de questões sociocientíficas, segundo Pedretti (2003, apud. MARTínEZ PÉREZ, 2012, p. 58), permitiu 
traçar um caminho concreto para alcançar os desafios do enfoque CTS, uma vez que a ciência e a tecnologia começaram a ser compreendidas como atividades humanas envolvidas em controvérsias e incertezas e que, portanto, exigem um posicionamento responsável dos cidadãos.

Em coerência com as palavras-chave empregadas nos artigos, os assuntos discutidos abordam, em várias ocasiões, questões sociocientíficas. No entanto, o tema mais destacado são as intervenções didáticas sobre CTS. A Tabela 4, apresenta os temas trabalhados e o número de ocorrências de cada tema. A partir da leitura completa dos textos, foi realizada a identificação dos temas, assim como a identificação dos objetos/sujeitos de pesquisa, dos tipos de metodologia empregados e dos problemas de pesquisa propostos.

Tabela 4. Temas abordados nas publicações.

\begin{tabular}{lc}
\multicolumn{1}{c}{ Tema } & $\begin{array}{c}\text { Número de } \\
\text { ocorrências }\end{array}$ \\
\hline Análise de uma Intervenção didática CTS & 10 \\
CTS e currículo & 3 \\
Controvérsias sociocientíficas na formação de professores & 2 \\
Mediações da fotografia para abordar Questões socioambientais & 2 \\
Percepções dos professores sobre CTS & 2 \\
A argumentação em discussões sociocientíficas & 1 \\
Ensino-Aprendizagem baseado em Questões sóciocientíficas & 1 \\
Aspectos éticos e socioculturais em obras de ficção científica & 1 \\
CTS e consumismo & 1 \\
CTS no Brasil & 1 \\
Documentários como subsídio para CTS & 1 \\
Educação ambiental e temas controversos & 1 \\
Educação para a Sustentabilidade & 1 \\
Marx como referencial para CTS & 1 \\
O Júri simulado para promoção da argumentação & 1 \\
Relações entre justiça ambiental, ensino de ciências e cidadania & 1 \\
\hline Total & 30 \\
\hline
\end{tabular}

Do total de 16 temas abordados nos 30 artigos, sobressai o tema Análise de uma Intervenção didática CTS, com 10 ocorrências, ou seja, 33,3\% dos trabalhos. Esse dado sugere que pesquisadores da área têm mostrado interesse na realização de atividades com enfoque CTS em períodos relativamente curtos e definidos.

É possível que essa marcada tendência à implementação e análise de intervenções didáticas corresponda à busca de um "método" determinado que permita, de forma processual ou sistemática, a inserção da perspectiva CTS na escola. Ideia que se afasta dos pressupostos de contextualização, problematização e participação do enfoque, pois existem inúmeras maneiras de realizar tal inserção, mas todas elas dependem do espaço/tempo de realização e, obviamente, dos interesses e as expectativas dos envolvidos. 
Também é possível que a medida esteja sendo implementada como alternativa diferenciada em contraposição às limitações de tempo e espaço para a realização de estudos CTS na organização escolar tradicional. Uma intervenção didática, de tipo "unidade" ou "sequência" (como é apresentado nos artigos analisados) pode ser vista como uma ação prudente e segura que, sem necessidade de modificar substancialmente o currículo, é capaz de permitir uma articulação entre os pressupostos do enfoque CTS e as exigências acadêmicas estabelecidas tradicionalmente, exigências que ocasionam serias dificuldades para os professores que pretendem trabalhar sob esse enfoque.

Porém, as dificuldades de inserção do enfoque vão além da organização escolar, estão relacionadas à identidade dos professores e a sua formação. Pedretti et al. (2006) e Aikenhead (2009) apontam algumas das situações que determinam a resistência dos professores com a implementação dos estudos CTS nas instituições escolares: preocupação por perder o "controle" da sala de aula; a falta de apoio da comunidade escolar que diminui o sentimento de pertencimento à escola; a politização em sala de aula que revela as concepções ideológicas dos professores; o compromisso com a formação pré-profissional, ou seja, a necessidade de formação meramente disciplinar e preparatória para o ingresso em níveis educacionais mais avançados; a falta de matérias de ensino; a falta de familiaridade com métodos de trabalho centrados no aluno (trabalhos em grupo, por exemplo); dificuldade para fazer relações entre linguagem cotidiana e científica; incerteza do papel do professor em sala de aula; dependência do livro didático; incerteza de como avaliar os alunos; conhecimentos e experiências de base inadequada (formação acadêmicoprofissional); problemas para trabalhar com questões políticas, históricas e filosóficas, entre outras.

Retomando os resultados do estudo, a Tabela 5, apresenta os Objetos/Sujeitos de pesquisa sobre os quais recaíram as análises dos autores. Os dados obtidos nesta etapa foram classificados em categorias para uma melhor interpretação das informações deles decorrentes. Essas categorias foram propostas pelo pesquisador (primeiro autor deste estudo) com base na semelhança de conteúdo ou de significado das informações analisadas.

É possível perceber que a maioria dos trabalhos (60\%) focou seus esforços na análise de diversos atores da comunidade acadêmica, ou seja, estudantes e professores de diferentes níveis do sistema educacional. A partir do reconhecimento dos temas apresentados anteriormente e com base na leitura dos documentos, foi possível verificar que nessas análises foram discutidos assuntos como: as percepções dos acadêmicos sobre temas relacionados com ciência e tecnologia; as capacidades de alunos e professores para realizar atividades com enfoque CTS e; os impactos cognitivos e comportamentais resultantes dessas atividades. Fica ressaltado o fato de vários desses trabalhos terem sido realizados com foco na educação básica, pois isto demostra que o enfoque CTS está abrindo, efetivamente, um espaço de trabalho prático na escola se considerarmos que 
autores como Pedretti et al. (2006) apontaram fortes avanços teóricos nos últimos anos, porém, poucos avanços na prática.

Tabela 5. Objetos/Sujeitos de estudo pesquisados.

\begin{tabular}{|c|c|c|c|}
\hline Tipo & Objeto/Sujeito de estudo & $\begin{array}{l}\text { Número de } \\
\text { ocorrências }\end{array}$ & $\begin{array}{l}\text { \% ocorrências } \\
\text { por categoria }\end{array}$ \\
\hline \multirow{8}{*}{$\begin{array}{l}\text { Comunidade } \\
\text { acadêmica }\end{array}$} & Alunos de ensino médio & 4 & \multirow{8}{*}{60} \\
\hline & Alunos de licenciatura & 3 & \\
\hline & Alunos do ensino fundamental & 3 & \\
\hline & Alunos de ensino médio e seus professores & 2 & \\
\hline & Professores de educação básica & 2 & \\
\hline & Professores de ensino médio & 2 & \\
\hline & Alunos de graduação & 1 & \\
\hline & Alunos de mestrado em educação & 1 & \\
\hline \multirow{5}{*}{$\begin{array}{l}\text { Produção } \\
\text { científica }\end{array}$} & Livros & 2 & \multirow{5}{*}{20} \\
\hline & Artigos sobre CTS & 1 & \\
\hline & Atas de eventos na área de ensino & 1 & \\
\hline & Marco teórico educacional STEPWISE & 1 & \\
\hline & Teorias sobre EC e EA & 1 & \\
\hline \multirow{4}{*}{$\begin{array}{l}\text { Produção } \\
\text { didática }\end{array}$} & Unidades didáticas & 1 & \multirow{4}{*}{13,3} \\
\hline & $\begin{array}{l}\text { Experiências de ensino em contextos de } \\
\text { vulnerabilidade socioambiental }\end{array}$ & 1 & \\
\hline & Disciplina de Química orgânica aplicada & 1 & \\
\hline & Produção discursiva de dois júris simulados & 1 & \\
\hline $\begin{array}{c}\text { Documentos } \\
\text { oficiais }\end{array}$ & Diretrizes curriculares & 1 & 3,3 \\
\hline Outros & Filmes documentários & 1 & 3,3 \\
\hline \multicolumn{2}{|l|}{ Total } & 30 & 100 \\
\hline
\end{tabular}

A respeito dos problemas de pesquisa levantados nas publicações, apenas 8 artigos (26,7\%) detalham seu problema de forma explicita por meio de pergunta ou questão de pesquisa, nos outros 22 artigos (73,3\%) o problema de pesquisa está implícito. O Quadro 2, expõe o texto das perguntas formuladas.

As questões de pesquisa apresentadas reiteram a intenção dos pesquisadores de conhecer e compreender as percepções de estudantes e professores acerca de assuntos relacionados com desenvolvimento tecnocientífico, e também de conhecer quais as dificuldades e potencialidades da implementação de atividades sob a perspectiva CTS.

Com o intuito de conhecer como procederam os autores para tratar seus problemas de pesquisa, foi realizada uma caracterização dos tipos de metodologias por eles utilizadas. A classificação acompanhou o tipo de abordagem, os objetivos de estudo e os procedimentos empregados, encontrando que a maioria das produções se apoiaram em análises qualitativas, tiveram como base a descrição dos fenômenos estudados e optaram por estudos de caso. A tabela 6, mostra os resultados dessa classificação. 
Quadro 2. Questões de pesquisa propostas.

\begin{tabular}{|c|c|}
\hline $\begin{array}{l}\text { "É possível integrar numa proposta curricular para a Educação Profissional } \\
\text { e Tecnológica (EPT) os pressupostos do campo Ciência, Tecnologia e } \\
\text { Sociedade (CTS) com os do campo Trabalho e Educação (T\&E)?" }\end{array}$ & ARAÚJO; SILVA, 2012. \\
\hline $\begin{array}{l}\text { "Como professores, em formação inicial, posicionam-se frente à } \\
\text { possibilidade de realizar encaminhamentos curriculares pautados pela } \\
\text { abordagem temática, durante seu estágio curricular e/ou no decorrer da } \\
\text { sua atuação profissional?" }\end{array}$ & ROSO et al., 2015. \\
\hline $\begin{array}{l}\text { "Uma sequência didática com o tema agrotóxicos numa perspectiva CTS } \\
\text { contribui para a reflexão dos alunos acerca de questões socioambientais?" }\end{array}$ & $\begin{array}{l}\text { BUFFOLO; RODRIGUES } \\
2015 .\end{array}$ \\
\hline $\begin{array}{l}\checkmark \text { "Que dificuldades são sentidas pelos professores na conceção e } \\
\text { implementação de atividades de cariz CTS? E quais as suas vantagens?" } \\
\checkmark \text { "Quais as perceções dos professores relativamente à metodologia de } \\
\text { ensino CTS?" }\end{array}$ & $\begin{array}{l}\text { BETTENCOURT; } \\
\text { ALBERGARIA-ALMEIDA; } \\
\quad \text { VELHO, } 2014 \text {. }\end{array}$ \\
\hline $\begin{array}{l}\text { "In what ways do students demonstrate identities as environmentally } \\
\text { literate persons (ELPS) in the Heat Game?" } \\
\checkmark \text { "In what ways do students demonstrate awareness of the connections } \\
\text { between science, technology, and the environment and awareness of } \\
\text { the consequences of the choices we make?" }\end{array}$ & REES, 2014. \\
\hline $\begin{array}{l}\text { "Quais as dificuldades e potencialidades inerentes ao planejamento e } \\
\text { aplicação de uma proposta de educação alimentar para estudantes do } \\
\text { ensino médio orientada segundo princípios defendidos pelo Movimento } \\
\text { CTS?" }\end{array}$ & $\begin{array}{l}\text { SANTANA; BASTOS; } \\
\text { TEIXEIRA, } 2015 \text {. }\end{array}$ \\
\hline $\begin{array}{l}\text { "Que evidências indicam contribuições e limitações da proposta de } \\
\text { formação analisada?" }\end{array}$ & $\begin{array}{l}\text { SANTOS; SILVA; } \\
\text { PEDROSA, } 2015 .\end{array}$ \\
\hline $\begin{array}{l}\text { "Em que medida as atividades de pesquisa pautadas em aspectos CTSA } \\
\text { que foram trabalhadas junto a alunos do Ensino Médio proporcionam } \\
\text { melhor compreensão dos elementos teóricos da perspectiva do Ensino por } \\
\text { Pesquisa?" }\end{array}$ & $\begin{array}{c}\text { RIBEIRO; GENOVESE, } \\
2015 .\end{array}$ \\
\hline
\end{tabular}

Tabela 6. Tipos de metodologias empregadas.

\begin{tabular}{clc}
\hline \multicolumn{2}{c}{ Tipos de metodologias } & $\begin{array}{c}\text { Número de } \\
\text { Ocorrências }\end{array}$ \\
\hline \multirow{2}{*}{ Quanto à abordagem } & Qualitativa & 28 \\
& Quantitativa & 2 \\
\hline \multirow{3}{*}{ Quanto aos objetivos } & Descritiva & 26 \\
& Exploratória & 3 \\
& Explicativa & 1 \\
\hline \multirow{4}{*}{ Quanto aos procedimentos } & Estudo de caso & 16 \\
& Investigação-ação & 4 \\
& Bibliográfica & 4 \\
& Levantamento & 3 \\
& Documental & 2 \\
& Etnográfica & 1 \\
\hline
\end{tabular}

Relacionando os tipos de metodologias empregadas, os sujeitos de estudo e os problemas de pesquisa indicados, é razoável pensar que os estudos de caso são uma estratégia pertinente para analisar as pequenas intervenções com enfoque CTS realizadas 
nos diferentes níveis acadêmicos, possibilitando a descrição dos processos e os resultados dessas ações.

Com o propósito de conhecer como foi estruturado o corpus teórico sobre CTS no país durante os últimos anos, foi realizada uma listagem dos autores mais citados. Os resultados (Tabela 7) denotam a instauração de um diálogo entre autores estrangeiros e brasileiros, com predominância dos nacionais. Esse dado, em concordância com Abreu, Fernandes e Martins (2013), aponta para a consolidação de um conjunto de referentes brasileiros que discutem o tema, gerando, consequentemente, uma forma particular de pensar o enfoque CTS. Os aportes de autores como Wildson Santos, Eduardo Mortimer, Décio Auler e Demétrio Delizoicov consistem, primordialmente, na aproximação dos estudos CTS com a perspectiva humanística de Educação proposta por Paulo Freire (AULER; DELIZOICOV, 2006; AULER et al., 2009; SANTOS; MORTIMER, 2002; SANTOS, 2008).

Tabela 7. Autores mais citados nos artigos analisados.

\begin{tabular}{|c|c|c|}
\hline Autor & País de atuação & Número de citações \\
\hline SANTOS, W. L. P. & Brasil & 39 \\
\hline AULER, D. & Brasil & 22 \\
\hline AIKENHEAD, G. & Canadá & 18 \\
\hline REIS, P. & Portugal & 14 \\
\hline SADLER, T. D. & EUA & 13 \\
\hline ACEVEDO DÍAZ, J. A. & Espanha & 11 \\
\hline MARTINS, I.P. & Portugal & 10 \\
\hline BAZZO, W. A. & Brasil & 8 \\
\hline GALVÃO, C. & Portugal & 8 \\
\hline YAGER, R. E. & EUA & 8 \\
\hline DELIZOICOV, D. & Brasil & 7 \\
\hline GIL PÉREZ, D. & Espanha & 7 \\
\hline LEVINSON, R. & Inglaterra & 7 \\
\hline ZIMAN, J. & Inglaterra & 7 \\
\hline CACHAPUZ, A. & Portugal & 6 \\
\hline FOUREZ, G. & Bélgica & 6 \\
\hline FREIRE, P. & Brasil & 6 \\
\hline KOLST Ø, S. D. & Noruega & 6 \\
\hline KRASILCHICK, M. & Brasil & 6 \\
\hline MORTIMER, E. F. & Brasil & 6 \\
\hline ZEIDLER, D. L. & EUA & 6 \\
\hline VIEIRA, R. D. & Brasil & 6 \\
\hline BENCZE, J.L. & Canadá & 5 \\
\hline GEE, J.P. & EUA & 5 \\
\hline SIMONNEAUX, L. & França & 5 \\
\hline
\end{tabular}

No entanto, parece existir ainda uma dependência teórica de produções estrangeiras, principalmente dos Estados Unidos e da Europa. Essa situação pode ser considerada 
previsível, pois conforme relata López Cerezo (1998), historicamente foram consideradas duas grandes tradições nos estudos CTS, a europeia, que pretendia estudar e problematizar os antecedentes sociais que determinam o desenvolvimento científico e tecnológico, e a norte-americana, cujo foco era analisar as consequências sociais e ambientais da produção científica em conjunto com os fatores éticos imersos no avanço tecnocientífico.

Tabela 8. Obras mais citadas.

\begin{tabular}{|c|c|}
\hline OBRA & $\begin{array}{l}N^{\circ} \text { de } \\
\text { Citações }\end{array}$ \\
\hline $\begin{array}{l}\text { AIKENHEAD, G. What is STS science teaching? In: SOLOMON, J.; AIKENHEAD, G. (Ed.). } \\
\text { STS education: international perspectives on reform. New York: Teachers College Press, } \\
\text { 1994. p. 47-59. }\end{array}$ & 7 \\
\hline $\begin{array}{l}\text { AULER, D. Alfabetização científico-tecnológica para quê? Ensaio: pesquisa em educação } \\
\text { em ciências, Belo Horizonte, v. 3, n. 2, p. 12-15, } 2001 .\end{array}$ & 7 \\
\hline $\begin{array}{l}\text { BOGDAN, R.; BIKLEN, S. Investigação qualitativa em educação: uma introdução à } \\
\text { teoria e aos métodos. Porto: Porto Editora, } 2010 .\end{array}$ & 6 \\
\hline $\begin{array}{l}\text { FOUREZ, G. Crise no ensino de ciências? Investigações em Ensino de Ciências, Porto } \\
\text { Alegre, v. 8, n. 2, p. 109-123, ago. } 2003 .\end{array}$ & 5 \\
\hline FREIRE, P. Pedagogia do oprimido. 47. ed. São Paulo: Paz e Terra, 2005. & 4 \\
\hline $\begin{array}{l}\text { LEVINSON, R. (2006). Towards a Theoretical Framework for Teaching Controversial } \\
\text { Socio-scientific Issues. International Journal of Science Education, } 28 \text { (10), p. 1201-1224. }\end{array}$ & 4 \\
\hline $\begin{array}{l}\text { MARTINS, I. P. Problemas e perspectivas sobre a integração CTS no sistema educativo } \\
\text { português. Revista Electrónica de Enseñanza de las Ciencias, Barcelona, v. 1, n. 1,p. 28- } \\
\text { 39, 2002. }\end{array}$ & 4 \\
\hline $\begin{array}{l}\text { PINHEIRO, N. A. M., SILVEIRA, R. M. C. F. \& BAZZO, W. A. (2007). Ciência, tecnologia e } \\
\text { sociedade: a relevância do enfoque CTS para o contexto do ensino médio. Ciência \& } \\
\text { Educação, Bauru, 13(7), 71-84. }\end{array}$ & 4 \\
\hline $\begin{array}{l}\text { SADLER, T. D. (2004). Informal Reasoning Regarding Socioscientific Issues: A Critical } \\
\text { Review of Research. Journal of Research in Science Teaching, } 41 \text { (5), p. 573-536. }\end{array}$ & 4 \\
\hline $\begin{array}{l}\text { SANTOS, W. L. P. dos, \& MORTIMER. E. F. (2000). Uma análise de pressupostos teóricos } \\
\text { da abordagem C-T-S (Ciência-Tecnologia Sociedade) no contexto da educação } \\
\text { brasileira. Ensaio: Pesquisa em Educação em Ciências, } 2 \text { (2), p. 133-162. }\end{array}$ & 3 \\
\hline $\begin{array}{l}\text { SANTOS, W. L. P. dos. Educação científica humanística em uma perspectiva Freireana: } \\
\text { resgatando a função do ensino de CTS. Alexandria: Revista de Educação em Ciência e } \\
\text { Tecnologia, Florianópolis, v. } 1, \text { n. 1, p. 109-137, 2008. }\end{array}$ & 3 \\
\hline $\begin{array}{l}\text { SANTOS, W. L. P., \& SCHNETZLER, R. P. (1997). Educação em química: compromisso } \\
\text { com a cidadania. Ijuí: Unijuí. }\end{array}$ & 3 \\
\hline $\begin{array}{l}\text { SILVA, L. F.; CARVALHO, L. M. A Temática Ambiental e o Processo Educativo: O ensino } \\
\text { de física a partir de temas controversos. Ciência \& Ensino. Vol.7, No Esp. }\end{array}$ & 3 \\
\hline $\begin{array}{l}\text { ZEIDLER, D. L. et al. Beyond STS: a research based framework for socioscientific issues } \\
\text { education. Science Education, Hoboken, v. 89, n. 3, p. 357-377, } 2005 .\end{array}$ & 3 \\
\hline $\begin{array}{l}\text { ZIMAN, J. The rationale of STS education is in the approach. In: SOLOMON, J.; } \\
\text { AIKENHEAD, G. (EdS.). STS education: international perspectives on reform. New York: } \\
\text { Teachers College Press, 1994. p. 27-31. }\end{array}$ & 3 \\
\hline
\end{tabular}

Em contrapartida, chama a atenção a falta de referências de outros autores latinoamericanos além dos brasileiros. Aparentemente, as produções sobre abordagem CTS em processos educacionais realizadas no Brasil, têm dialogado pouco com autores dos países 
vizinhos, quiçá pelas dificuldades de acesso às produções dos diferentes países, mas ignorando a importante necessidade da construção de uma identidade coletiva na América Latina. Isto é, uma identidade que considere o contexto territorial e cultural da região, entendendo que é preciso reestruturar o nosso modelo de progresso, buscando promover o respeito dos recursos naturais e humanos, procurando a diminuição da desigualdade social e permitindo a recuperação/criação da própria cultura do povo latino-americano.

Para finalizar esta seção, na tabela 8 aparecem as obras mais citadas nos artigos, com suas respetivas referências bibliográficas. Os 15 trabalhos mais referenciados podem ser considerados como leitura fundamental, não apenas para a compreensão dos pressupostos do enfoque CTS, mas como importante subsídio para a proposição de novos estudos e pesquisas na área da Educação.

\section{Algumas considerações}

O estudo realizado permitiu construir uma noção de como está sendo desenvolvido o cenário de pesquisa à luz do enfoque CTS durante os últimos anos no Brasil. A partir da leitura e caracterização do conteúdo dos artigos publicados em revistas especializadas em Educação científica, foram identificados os principais temas de estudo, os problemas de pesquisa estudados e os procedimentos metodológicos realizados, assim como os principais autores citados e as obras mais referenciadas.

Esses resultados permitem pensar que, embora a produção na perspectiva CTS seja pouca em comparação com a produção total das áreas de Ensino e Educação, aconteceram avanços importantes na quantidade e qualidade de trabalhos realizados, criando um conjunto de produções que descrevem características da concepção desse enfoque, no país. Além disso, são notórios os alcances desse tipo de trabalho, atingindo diferentes agentes e níveis da vida acadêmica, promovendo a junção entre o trabalho prático e a produção teórica.

Desse modo, observou-se a simpatia dos autores pela realização de estudos de caso, principalmente de intervenções didáticas, em que analisaram as percepções, habilidades e dificuldades de estudantes e professores na implementação acadêmica do enfoque CTS, criando insumos para a realização de novos trabalhos e, consequentemente, a possibilidade de estabelecer novas perspectivas e novos rumos para o campo da Educação em Ciência e Tecnologia.

Percebe-se, contudo, a imperiosa necessidade de aproximar os estudos CTS realizados no Brasil com produções de outros países da América Latina, pois as nações da região, que têm sofrido historicamente as mesmas consequências do empreendimento tecnocientífico descontrolado, podem, por meio da construção de um pensamento coletivo sobre as interrelações entre ciência, ambiente, tecnologia e sociedade, criar o ponto de partida para mudar a forma de ver/entender o mundo e, consequentemente, propor um novo modelo de progresso social. 


\section{Referencias}

ABREU, T.B., FERNANDES, J.P., MARTINS, I. Levantamento Sobre a Produção CTS no Brasil no Período de 1980-2008 no Campo de Ensino de Ciências. Alexandria: Revista de Educação em Ciência e Tecnologia, v.6, n.2, p. 3-32, 2013.

AIKENHEAD, G. Research into STS science education. Revista Brasileira de Pesquisa em Educação em Ciências, v. 9, n. 1, p. 1-21, 2009.

ARAÚJO, A. B.; SILVA, M. A. Ciência, Tecnologia e Sociedade; Trabalho e Educação: possibilidades de integração no currículo da Educação Profissional e Tecnológica. Ensaio Pesquisa em Educação em Ciências, v. 14, n. 1, p. 99 -112, 2012.

AULER, D.; BAZZO, W. A. Reflexões para a implementação do movimento CTS no contexto educacional brasileiro. Ciência \&amp; Educação (Bauru), v. 7, n. 1, p. 1-13, 2001.

AULER, D.; DALMOLIN, A. M. T.; FENALTI, V. Abordagem temática: natureza dos temas em Freire e no enfoque CTS. Alexandria: Revista de Educação em Ciência e Tecnologia, v. 2, n.1, p. 67-84, 2009.

AULER, D.; DELIZOICOV, D. Educação CTS: Articulação entre pressupostos do educador Paulo Freire e referenciais ligados ao movimento CTS. In: LÓPES, A. B.; PEINADO, V-B.; LÓPES, M. J.; RUZ, M. T. P. (Org.). Las Relaciones CTS en la Educación Científica. Málaga: Editora da Universidade de Málaga, v. Único, p. 01-07, 2006.

AZEVEDO, R. et al. Questões sociocientíficas com enfoque CTS na formação de professores de Ciências: perspectiva de complementaridade. Amazônia: Revista de Educação em

Ciências e Matemáticas, v. 9, n. 18, p. 84-98, jun. 2013.

BETTENCOURT, C.; ALBERGARIA-ALMEIDA, P.; VELHO, J. L. Implementação de Estratégias Ciência-Tecnologia-Sociedade (CTS): perceções de professores de biologia. Investigações em Ensino de Ciências, v. 19, n. 2, p. 243-261, 2014.

BUFFOLO, A.C.C; RODRIGUES, M. A. Agrotóxicos: uma proposta socioambiental reflexiva no ensino de Química sob a perspectiva CTS. Investigações em Ensino de Ciências (Online), v. 20, p. 1-14, 2015.

CAPES, Plataforma Sucupira, disponível em:

<http://www.capes.gov.br/avaliacao/plataforma-sucupira> 2017a.

CAPES, Plataforma Sucupira, disponível em:

<https://sucupira.capes.gov.br/sucupira/public/index.xhtml> 2017b.

COCENZA, A.; MARTINS, I. Controvérsias Socioambientais no Contexto da Construção de Sentidos Sobre Relações Entre Energia e Ambiente na Escola. Revista Brasileira de Pesquisa em Educação em Ciências, v. 13, p. 73-94, 2013. 
LÓPEZ CEREZO, J. A. Ciencia, Tecnología y Sociedad: el estado de la cuestión en Europa y Estados Unidos. Revista Iberoamericana de Educación, v. 18, p. 41-68, 1998.

MARTíNEZ ÁLVAREZ, F. El Movimiento de Estudios Ciencia- Tecnología- Sociedad: su origen y tradiciones fundamentales. Humanidades Médicas, v. 4, n. 1, p. 0-0, 2004.

MARTíNEZ PÉREZ, L.F. Cuestiones sociocientíficas en la formación de profesores de ciencias: aportes y desafíos. Revista de la Facultad de Ciencia y Tecnología, n. 36, p. 77-94, 2014.

MARTÍNEZ PÉREZ, L.F. Questões sociocientíficas na prática docente Ideologia, autonomia e formação de professores. São Paulo: Editora Unesp, 2012.

MATTOS, C. R.; VILLANI, A. Apresentação: Environmental Discourses in Science Education: contributions to democracy, citizenship and social justice. Revista Brasileira de Pesquisa em Educação em Ciências, v. 14, n. 2, 2014.

MENDES, M. R. M.; SANTOS, W. L. P. dos. Argumentação em discussões sociocientíficas. Investigações em Ensino de Ciências (Online), v. 18, p. 621-643, 2013.

MEMBIELA, P. Una Revisión del Movimiento Educativo Ciencia-Tecnología-Sociedad. Enseñanza de las Ciencias, Investigación y Experiencias Didácticas., v. 15, n. 1, p. 51-57, 1997. MUHR, T. ATLAS/ti - A Prototype for the Support of Text Interpretation. Qualitative Sociology, v. 14, n. 4, 1991.

PEDRETTI, E. G.; BENCZE, L.; HEWITT, J.; ROMKEY, L.; JIVRAJ, A. Promoting Issues-based STSE Perspectives in Science Teacher Education: Problems of Identity and Ideology. Science and Education, v. 17, n. 8/9, p. 941-960, 2006.

QUINTERO CANO, C. A. Enfoque Ciencia, Tecnología y Sociedad (CTS): perspectivas educativas para Colombia. Zona Próxima, n. 12, p. 222-239, 2010.

REES, C. Developing Awareness of Connections Between Science, Technology and the Environment through Participation in a Game-Like Approach to Curriculum, Revista Brasileira de Pesquisa em Educação em Ciências, v. 14, n. 2, p. 147-157, 2014.

RIBEIRO, T.; GENOVESE, L. O emergir da perspectiva de Ensino por Pesquisa de Núcleos Integrados no contexto da implementação de uma proposta CTSA no Ensino Médio. Ciência \& Educação, v. 21, p. 1-29, 2015.

ROSO, C; SANTOS, R; ROSA, S; AULER, D. Currículo Temático fundamentado em Freire-CTS: Engajamento de professores de Física em formação inicial. Ensaio: Pesquisa em Educação em Ciências (Online), v. 17, p. 372-389, 2015.

SANTANA, T. A.; BASTOS, A.P.S.; TEIXEIRA, P.M.M. Nossa alimentação: Análise de uma sequência didática estruturada segundo referenciais do Movimento CTS. Revista Brasileira de Pesquisa em Educação em Ciências, v. 15, p. 105-122, 2015. 
SANTOS, L.; SILVA, R. M. G.; PEDROSA, M. A. Formação em Educação para o Desenvolvimento Sustentável: um estudo com professores de Ciências da Natureza. Revista brasileira de Pesquisa em Educação em Ciências, v. 15, p. 357-386, 2015.

SANTOS, W. L. P. Educação Científica Humanística em uma perspectiva Freireana: resgatando a função do ensino de CTS. Alexandria: Revista de Educação em Ciência e Tecnologia, v. 1, n. 1, p. 109-131, 2008.

SANTOS, W. L. P. Educação CTS e cidadania: confluências e diferenças. Amazônia: Revista de Educação em Ciências e Matemáticas, v. 9, n. 17, p. 49-62, dez. 2012.

SANTOS, W. L. P.; MORTIMER, E. F. Uma análise de pressupostos teóricos da abordagem CT-S (Ciência - Tecnologia - Sociedade) no contexto da educação brasileira. Ensaio Pesquisa em Educação em Ciências, v. 2, n. 2, p. 1-23, 2002.

STRIEDER, R.B. Abordagem CTS na Educação Científica no Brasil: sentidos e perspectivas. Tese (Doutorado em Ensino de Ciências). Programa de Pós-Graduação Interunidades em Ensino de Ciências, Universidade de São Paulo, São Paulo, 2012. 